\title{
Ensino de Música apoiado pelo uso de serious games: revisão sistemática sobre o panorama de publicações nacionais e internacionais
}

\author{
Victor A. Freitas ${ }^{1}$, Alana M. de Morais ${ }^{2}$ \\ ${ }^{1}$ Programa de Pós-Graduação em Computação, Comunicação e Artes (PPGCCA) \\ Centro de Informática, Universidade Federal da Paraíba (UFPB), 58051-900 - \\ João Pessoa - PB, Brasil \\ ${ }^{2}$ Coordenação de Sistemas - Instituto de Educação Superior da Paraíba - IESP \\ Cabedelo - PB, Brasil \\ tito.victor@hotmail.com, alanam2@gmail.com
}

\begin{abstract}
The Music teaching by digital games has resulted in an improvement in the quality of learning and the instructional efficiency. These applications have many goals, such as: motivating students, supporting teaching, monitoring students, and evaluating the student or classroom. The current article aims to present a systematic review about researches that use digital educational games (or serious games) to support the Music teaching. For this, articles published in journals and events (international and Brazilian events) were selected to answer how the academy is discussing this thematic. The results point out an evolution of studies focused on children music education, offering support for the introduction of music in the school. Besides that, only two research propose the teaching of musical elements for adults.
\end{abstract}

Resumo. O ensino de Música apoiado pelo uso de jogos digitais vem resultando em uma melhoria na qualidade da aprendizagem e uma maior eficiência instrucional. Esses aplicativos possuem intuitos distintos, tais como: motivar discentes, apoiar o ensino, monitorar estudantes e até mesmo avaliar o aluno ou a turma. O presente artigo tem como objetivo apresentar uma revisão sistemática de trabalhos acadêmicos que utilizam jogos digitais educativos (ou jogos sérios - serious games) para apoiar o ensino de conteúdos relacionados à Música. Para isso, foram selecionados trabalhos publicados em conferências, revistas nacionais e internacionais a fim de entender e analisar como a academia está discutindo sobre a temática. Os resultados apontam uma evolução nas pesquisas com objetivo em educação musical para crianças, oferecendo apoio a introdução na área de Música no contexto escolar e apenas duas pesquisas propõem o ensino de elementos musicais para adultos.

\section{Introdução}

A escola é o local em que o estudante inicia sua jornada de conhecimento, na busca pelo aprendizado alguns alunos acabam conhecendo a área de Música, descobrindo novas habilidades que, na maioria das vezes, acontecem por meio de aulas tradicionais. Os 
jogos sérios voltados para o ensino de Música estimulam os alunos a desenvolver habilidades intelectuais, práticas sem a necessidade de ter um instrumento musical e vivenciar novos ambientes que são inacessíveis para o formato tradicional de aula. Os alunos são estimulados e demonstram interesse em conhecer tudo aquilo que é apresentado, possibilitando assim, condições novas de aprendizado [Moreira e Castro, 2016].

Tendo em vista a importância dos jogos educacionais no ensino de Música, o presente trabalho propõe entender quais são as iniciativas sobre o ensino de Música apoiado por jogos sérios, analisar o estado da arte das pesquisas realizadas no mundo e investigar se as informações sobre tais pesquisas são relevantes e quais lacunas faltam ser preenchidas. Neste sentido, optou-se por realizar o processo de Revisão Sistemática (RS) com objetivo de investigar, mapear e discutir pesquisas voltadas para o ensino de Música por meio de jogos sérios.

A RS da literatura é uma metodologia de busca científica que permite identificar, avaliar e interpretar pesquisas disponíveis e relevantes para uma determinada questão de pesquisa ou área temática [Kitchenham, 2004]. Este método investigativo requer mais esforço do que investigações tradicionais [Biolchini et al., 2005]. Sua principal vantagem é fornecer informações sobre os efeitos de algum fenômeno por meio de uma ampla gama de configurações e métodos empíricos. Essa metodologia aumenta a probabilidade de detecção de efeitos reais que estudos individuais são incapazes de detectar. Portanto, o presente artigo apresenta uma RS da literatura baseado nos principais eventos e revistas da área de jogo sérios. O objetivo é identificar trabalhos acadêmicos que utilizam jogos sérios no ensino de Música, quais as áreas de Música são mais exploradas por estes softwares e como os jogos sérios são incorporados nas rotinas de ensino-aprendizagem de Música. Os resultados mostram que $53 \%$ dos estudos foram voltados para educação musical no contexto infantil e apenas $8 \%$ das pesquisas utilizaram jogos sérios para ensinar elementos musicais para adultos.

A fim de esclarecer o método científico utilizado e os resultados do estudo o presente artigo foi organizado em cinco seções principais. Na segunda seção, apresentam-se pesquisas acadêmicas cujos objetivos são ensino-aprendizagem por meio de jogos sérios. Na terceira seção deste artigo, tem-se o planejamento e execução da RS. A quarta seção contempla os principais resultados das investigações. Por fim, a quinta e última seção apresenta as considerações finais e os trabalhos futuros relacionados ao estudo.

\section{Trabalhos Correlatos}

A área de jogos sérios para ensino-aprendizagem apresenta uma série de trabalhos com conteúdo diversificado, tais como Educa Direito [Freitas e Adamatti, 2016] jogo criado para o ensino do direito do trabalho, LibrasZap [Sarinho, 2017] para ensino da Língua Brasileira de Sinais. Além de vários estudos para mostrar os processos de desenvolvimentos de jogos educacionais, como o "Estudo Bibliográfico sobre o Processo de Construção de Jogos Digitais: A Necessidade de Sinergia entre o Educar e o Divertir" [Cox e Bittencourt, 2017] e "Revisão sistemática, como jogos educacionais são desenvolvidos?" [Battistella et al., 2014]. Diante disso, todos os jogos educativos 
VIII Congresso Brasileiro de Informática na Educação (CBIE 2019)

Anais do XXX Simpósio Brasileiro de Informática na Educação (SBIE 2019)

ensinam algo de uma determinada área, mas são poucos os voltados para o ensino de Música.

Entretanto, alguns trabalhos utilizam uma metodologia semelhante ao artigo proposto, como exemplo, "Tecnologias no Ensino da Música: revisão integrativa de investigações realizadas no Brasil e em Portugal" [Fernandes e Coutinho, 2014], que tem como objetivo apresentar uma revisão dos artigos referentes à utilização de tecnologia para o ensino de Música. Outro trabalho relacionado à proposta de pesquisa é "Evidence-based Frameworks for Teaching and Learning in Classical Singing Training: A Systematic Review" [Crocco et al., 2017], que tem como objetivo apresentar uma RS de trabalhos acadêmicos publicados sobre canto clássico, outra área importante de Música. Ambas as pesquisas apresentam RS de estudos voltados para ensino de Música por meio de tecnologias, mas não foram encontrados trabalhos que solucionassem a mesma problemática do presente estudo, de acordo com as estratégias adotadas na RS.

\section{Metodologia da Revisão Sistemática}

Segundo a metodologia utilizada (Figura 1), são formuladas perguntas de pesquisas, selecionadas as bases para localização dos estudos, definidos os termos de busca, analisados os periódicos, executada uma triagem das buscas e apresentados os resultados da investigação.

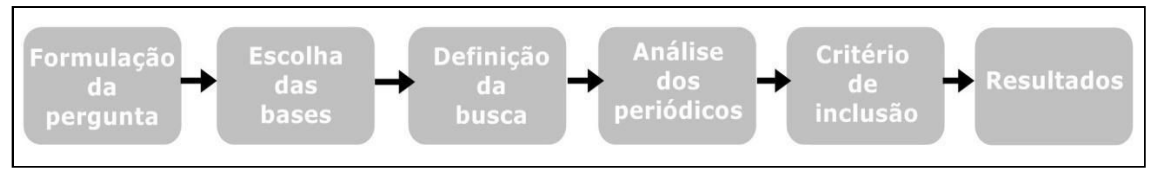

Figura 1. Estrutura da Revisão Sistemática

A etapa inicial da formulação da pergunta de pesquisa é fundamental para o planejamento e delineamento dos objetivos da RS. Neste sentido, algumas perguntas de pesquisa podem ser destacadas no presente trabalho, são elas: P1: Quais áreas de Música são as mais exploradas nos jogos sérios? P2: Como os jogos sérios estão sendo utilizados no ensino de Música?

\subsection{Definição da Busca}

Para realizar a investigação da produção científica e alcançar resultados significativos, foram escolhidas as seguintes bases de dados: ACM Digital Library (ACM), IEEExplorer (IEEE), Simpósio Brasileiro de Informática na Educação (SBIE), onde estão publicados boa parte dos conteúdos relacionados à Informática na Educação sendo realizado em paralelo com Congresso Brasileiro de Informática na Educação (CBIE), Revista Brasileira de Informática na Educação (RBIE) e a Scopus Elsevier (SCOPUS) que contém diversas bases incorporadas. O presente estudo definiu que serão analisados somente trabalhos publicados entre 2005 e 2019 nas bases anteriormente citadas.

\subsubsection{Termos de busca}

Os termos usados para execução da RS nas bases de dados ACM, IEEE, SBIE, RBIE e SCOPUS foram "(jogo musical) OR (jogos de música) OR (ensino de música) OR (music AND Game AND education OR Game AND music AND education)". Na 
VIII Congresso Brasileiro de Informática na Educação (CBIE 2019)

Anais do XXX Simpósio Brasileiro de Informática na Educação (SBIE 2019)

ACM, IEEE e SCOPUS apresentam em seus resultados publicações de outras bases não citadas, mas que foram consideradas dada a relevância das publicações.

\subsection{Análise dos periódicos}

A análise dos resultados, utilizando a chave de busca definida anteriormente, retornou um total de 353 artigos publicados na base SCOPUS, ACM retornou um total de 86 artigos, obtiveram-se 60 artigos resultantes na base IEEE, na SBIE resultou 8 artigos e na RBIE 5 artigos.

\subsubsection{Critério de Inclusão}

Os artigos obtidos passaram por um processo de filtragem. Nesse processo, são lidos e analisados o resumo, o título e, se necessário, a introdução do trabalho. Caso um dos critérios seja respeitado, automaticamente o trabalho é selecionado para a fase de leitura completa. Os critérios definidos para esta pesquisa foram:

Critério 1- Serão incluídos trabalhos que comparem ou avaliem instrumentos teóricos e/ou práticos de jogos sérios para o ensino-aprendizagem de assuntos voltados para áreas de Música.

Critério 2- Serão incluídos trabalhos que apresentem métodos de desenvolvimento de jogos sérios para o ensino-aprendizagem de Música.

Critério 3- Serão incluídos trabalhos que proponham um design de jogos sérios para ensino de Música.

Critério 4- Serão incluídos artigos que apresentem uma proposta interdisciplinar entre Música e Computação e discutam sobre o ensino de Música.

\subsubsection{Critério de Exclusão}

Critério 1- Serão excluídos trabalhos repetidos que apareçam em mais de uma base de busca, considerando apenas o resultado mais recente.

Critério 2- Serão excluídos trabalhos no formato de livro, resumos estendidos ou que não tiverem acesso por meio do periódicos Capes via acesso café ${ }^{1}$.

\section{Resultados}

Diferentes autores têm diversos conceitos sobre o termo jogos sérios ou educacionais [Salen e Zimmerman, 2004. Raziūnaite, P. et al, 2018]. Dessa forma, torna-se pertinente definir que jogo sério no presente trabalho como sistemas computacionais que tenham regras bem definidas, interatividade, gráficos $3 \mathrm{D}$ ou $2 \mathrm{D}$, objetivos claros, narrativas construídas em primeira ou terceira pessoa, componentes de Inteligência Artificial (IA) e elementos desafiantes ao jogador, como: pontuação, competição, tempo ou ranking.

Após a execução da busca nos periódicos definidos, todos os artigos resultantes passaram pelo processo de filtragem e os critérios de inclusão e exclusão foram

${ }^{1} \mathrm{O}$ acesso café habilita o acesso gratuito a todos os Discentes, Docentes e Técnicos Administrativos ao Portal de Periódico da Capes por meio da Comunidade Acadêmica Federada - CAFe, que permite o acesso dentro ou fora da universidade. 
VIII Congresso Brasileiro de Informática na Educação (CBIE 2019)

Anais do XXX Simpósio Brasileiro de Informática na Educação (SBIE 2019)

avaliados. O resultado da busca manual resultou em: 353 artigos selecionados na base SCOPUS, restando 9 artigos. Nessa mesma perspectiva, dos 86 artigos obtidos na base ACM foram incluídos 6 artigos, IEEE de 60 artigos restaram 12 artigos, na SBIE de 8 artigos apenas 3 e RBIE de 5 artigos restaram apenas 2 artigos.

Contudo, considerando a investigação proposta pela RS, à maioria das informações básicas extraídas dos artigos está no título e resumo, boa parte tem relação com o objetivo de ensino-aprendizagem de Música por meio de jogos sérios. Desse modo, a Figura 2 apresenta um panorama geral da busca realizada, no qual foram analisados os critérios de inclusão e exclusão, resultando no total de 25 artigos para fase da leitura completa.

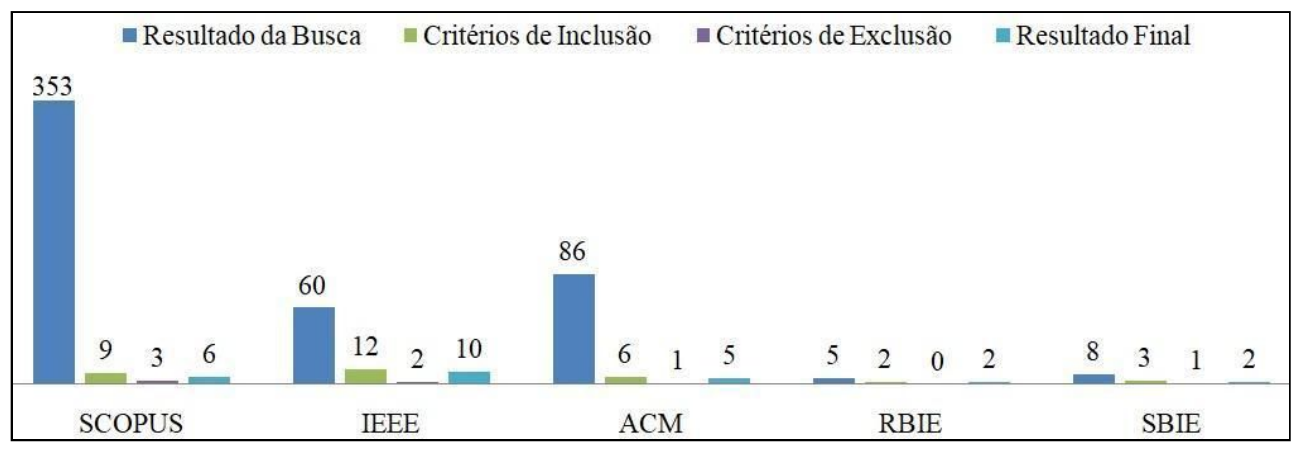

Figura 2. Resultado Final

Considerando os artigos incluídos no critério 1 como principal linha de pesquisa, estão os artigos cujo o número sequencial está presente na Tabela 1 são: A2, A5, A6, A7, A9, A11, A12, A13, A14, A15, A17, A19, A20, A21, A22, A23, A24, A25. No critério 2, encontram-se artigos relevantes, tais como A3 e A8. Nesse mesmo sentido, no critério 3, os artigos A4, A16 e A18 apresentaram design de jogos digitais para ensino de Música. Por fim, o critério 4 permitiu a inclusão dos artigos A1 e A10 com propostas interdisciplinar de Computação e Música. Além disso, foram excluídos dois artigos da SCOPUS por cobrança de taxa para obtenção do acesso ao arquivo, um resumo da ACM e quatro pesquisas repetidas, sendo duas da IEEE, uma da SCOPUS e uma da SBIE.

A Tabela 1 apresenta os 25 artigos resultantes do processo ordenados por um número sequencial (ID), título do artigo, a lista de autores e o ano de publicação. Além disso, as publicações foram agrupadas de acordo com a base de busca relacionada.

Tabela 1. Referência dos 25 artigos resultantes da avaliação dos critérios

\begin{tabular}{|c|c|c|c|c|}
\hline ID & Título & Autores & Base & Ano \\
\hline A1 & Girls, Music and Computer Science & $\begin{array}{c}\text { C. N. Silla, A. L. Przybysz, A. } \\
\text { Rivolli, T. Gimenez, C. Barroso, J. } \\
\text { Machado }\end{array}$ & IEEE & 2019 \\
\hline A2 & $\begin{array}{c}\text { Inclusive sound and music serious games in a } \\
\text { large-scale responsive environment }\end{array}$ & $\begin{array}{c}\text { M. Mandanici, F. Altieri, A. Rodà, } \\
\text { S. Canazza }\end{array}$ & scopus & 2018 \\
\hline A3 & $\begin{array}{c}\text { Designing an educational music game for } \\
\text { digital game based learning: A Lithuanian case } \\
\text { study }\end{array}$ & $\begin{array}{c}\text { P. Raziūnaitè, A. Miliūnaitè, R. } \\
\text { Maskeliūnas, R. Damaševičius, T. } \\
\text { Sidekerskienè, B. Narkevičienè }\end{array}$ & IEEE & 2018 \\
\hline A4 & $\begin{array}{c}\text { Experiencing musical rhythm through } \\
\text { interactive installation and AR/VR game } \\
\text { development }\end{array}$ & $\begin{array}{c}\text { S. Chung,H. Lin, T. Tsou,C. Wu,C. } \\
\text { Huang }\end{array}$ & 2018 \\
\hline
\end{tabular}


VIII Congresso Brasileiro de Informática na Educação (CBIE 2019)

Anais do XXX Simpósio Brasileiro de Informática na Educação (SBIE 2019)

\begin{tabular}{|c|c|c|c|c|}
\hline A5 & $\begin{array}{l}\text { Flappy crab project: Development of a digital } \\
\text { educational game for teaching Music }\end{array}$ & $\begin{array}{l}\text { C. M. C. Gomes, J. D. C. Gomes, } \\
\text { L. Oliveira }\end{array}$ & IEEE & 2017 \\
\hline A6 & $\begin{array}{l}\text { Integration of traditional music through mobile } \\
\text { game in inspiring Malaysian youths' enthusiasm }\end{array}$ & $\begin{array}{l}\text { F. Z. R. Azman, M. F. } \\
\text { Kamaruzaman }\end{array}$ & IEEE & 2017 \\
\hline A7 & $\begin{array}{c}\text { Development of a digital game-based learning } \\
\text { system in music learning }\end{array}$ & Y. Liang, Y. Shiue,Y. Huang,C. Liu & IEEE & 2017 \\
\hline $\mathrm{A} 8$ & $\begin{array}{l}\text { Designing an educational music game: What if } \\
\text { children were calling the tune? }\end{array}$ & $\begin{array}{l}\text { M. Nouwen, S. Schepers, K. } \\
\text { Mouws, K. Slegers, N. Kosten, P. } \\
\text { Duysburgh }\end{array}$ & SCOPUS & 2016 \\
\hline A9 & $\begin{array}{l}\text { Touching Notes: A Gesture-Based Game for } \\
\text { Teaching Music to Children }\end{array}$ & $\begin{array}{l}\text { M. Renzi, S. Vassos, T. Catarci, S. } \\
\text { Kimani }\end{array}$ & ACM & 2015 \\
\hline A10 & $\begin{array}{c}\text { Note Code: A Tangible Music Programming } \\
\text { Puzzle Tool }\end{array}$ & $\begin{array}{l}\text { V. Kumar, T. Dargan, U. Dwivedi, } \\
\text { P.i Vijay }\end{array}$ & ACM & 2015 \\
\hline A11 & E-cecilia: Implementation of a music game & $\begin{array}{c}\text { R. J. García, I. B. Pérez, J. } \\
\text { Lorenzo, T. García,J. Arambarri,M. } \\
\text { Milá , M. Sbert }\end{array}$ & SCOPUS & 2014 \\
\hline A12 & $\begin{array}{c}\text { Development of serious games for music } \\
\text { education }\end{array}$ & $\begin{array}{l}\text { A. Baratè, M. G. Bergomi, L. A. } \\
\text { Ludovico }\end{array}$ & SCOPUS & 2013 \\
\hline A13 & Music as a game controller & J. Heerema, J. R. Parker & IEEE & 2013 \\
\hline A14 & $\begin{array}{l}\text { E-Santur: A customizable tool for learning } \\
\text { Santur music instrument using game-based } \\
\text { learning approach }\end{array}$ & K. M. Yazdi, S. P. Lee & IEEE & 2011 \\
\hline A15 & $\begin{array}{l}\text { Pitch paradise: A mobile game as an } \\
\text { educational tool for music }\end{array}$ & $\begin{array}{l}\text { JR. Flores, J. Michael G. Sta. J. } \\
\text { Ma. R. C. Solamo, R. P. Feria }\end{array}$ & SCOPUS & 2011 \\
\hline A16 & $\begin{array}{c}\text { The application of educational game in } \\
\text { teaching the young children to read musical } \\
\text { notation }\end{array}$ & H. Ni , Z. Yang & IEEE & 2010 \\
\hline A17 & $\begin{array}{c}\text { The piano game: Using low cost laptops for } \\
\text { music education }\end{array}$ & $\begin{array}{l}\text { N. S. Patrício, I. K. Ficheman ,R. } \\
\text { de D. Lopes }\end{array}$ & SCOPUS & 2007 \\
\hline A18 & $\begin{array}{l}\text { Motivation-driven educational game design: } \\
\text { applying best practices to music education }\end{array}$ & Guillaume Denis, Pierre Jouvelot & $\mathrm{ACM}$ & 2005 \\
\hline A19 & $\begin{array}{l}\text { TuneTrain: Incidental learning through } \\
\text { interactive music creation }\end{array}$ & $\begin{array}{l}\text { J.Lee ,A.Gea,N. Levin,C. Yang,R. } \\
\text { Deciron,M. Lee, Pei-Lin Lu, M. Xie }\end{array}$ & IEEE & 2013 \\
\hline A20 & Chorlody: a music learning game & Y. Liu,N. Yan,Dili Hu & ACM & 2014 \\
\hline A21 & $\begin{array}{c}\text { Guitar man: (an implementation of a rhythm } \\
\text { game cooperative musical performance system } \\
\text { with actual musical instruments) }\end{array}$ & Aram So & ACM & 2008 \\
\hline A22 & $\begin{array}{l}\text { Genvirtual: Um jogo musical para reabilitação } \\
\text { de indivíduos com necessidades especiais }\end{array}$ & $\begin{array}{l}\text { A. G. D. Corrêa, G. A. de Assis, M. } \\
\text { do Nascimento,R. de D. Lopes }\end{array}$ & RBIE & 2008 \\
\hline A23 & $\begin{array}{l}\text { Zorelha: Um Objeto de Aprendizagem Para } \\
\text { Auxiliar o Desenvolvimento da Percepção } \\
\text { Musical em Crianças de } 4 \text { a } 6 \text { Anos }\end{array}$ & $\begin{array}{l}\text { E. A. de Jesus, M. Z. Uriarte, A. L. } \\
\text { A. Raabe }\end{array}$ & RBIE & 2010 \\
\hline A24 & $\begin{array}{l}\text { Musikinésia: Jogo Eletrônico para o } \\
\text { Aprendizado de Teclado Musical }\end{array}$ & $\begin{array}{l}\text { R. A. Bordini, J. L. Otsuka, D. M. } \\
\text { Beder, L. F. Fonseca, A. P. A. } \\
\text { Nunes, D. L. Santiago, P. A. G. de } \\
\text { Freitas, G. L. A. Santiago,M. R. G. } \\
\text { de Oliveira }\end{array}$ & SBIE & 2015 \\
\hline A25 & $\begin{array}{l}\text { Descobrindo Sons: um jogo para ensinar } \\
\text { música de forma lúdica }\end{array}$ & T. Napolitano, G. P. Guedes & SBIE & 2018 \\
\hline
\end{tabular}

\subsection{Panorama das publicações}

$\mathrm{Na}$ educação, dispor de jogos sérios como instrumento para ensino-aprendizagem denota um método dinâmico, interativo e motivador. Diante desses fatores, a realização 
VIII Congresso Brasileiro de Informática na Educação (CBIE 2019)

Anais do XXX Simpósio Brasileiro de Informática na Educação (SBIE 2019)

de um levantamento de jogos sérios e a relação com o ensino de Música é fundamental para este trabalho. Ao observar o ano dos artigos publicados, perceber-se que há poucas pesquisas na área de jogos sérios para ensino de Música, com um maior número de estudos acadêmicos sendo realizados no ano de 2018, conforme a Figura 3. Ao se observar a área de Música, nota-se uma resistência no uso de ferramentas digitais por professores em sala de aula, se comparado com o uso das tecnologias no dia a dia [ARAUJO; MORENO, 2009].

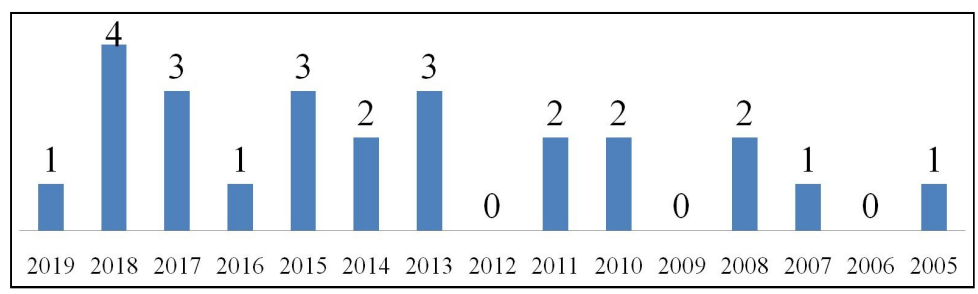

Figura 3. Ano das publicações

\subsubsection{Quais temáticas são as mais exploradas nos jogos sérios?}

As características encontradas, as variáveis de cada estudo e metodologias, são parâmetros considerados relevantes para a comparação dos estudos selecionados [Pocinho, 2008]. Neste sentido, destacam-se as principais temáticas abordadas no ensino de Música: Programação e Música, Elementos Musicais e Educação Musical, ilustrados pela Figura 4. Desse modo, é possível perceber que boa parte das publicações catalogadas foca em abordar uma introdução a educação musical por meio de jogos sérios (A3, A5, A6, A8, A9, A14, A15, A17, A19, A20, A21, A22, A23). Os artigos A2, A4, A7, A11, A12, A13, A16, A18, A24 e A25 focaram em um escopo voltado para elementos musicais como ritmo, melodia, harmonia, timbre, solfejo e por fim os artigos A1 e A10 abordaram assuntos de Programação e Música.

Outra característica é o público-alvo dos projetos desenvolvidos e selecionados. Entre os principais públicos estão as crianças (presentes nas pesquisas A2, A3, A5, A7, A8, A9, A10, A11, A15, A16, A17, A19, A22 e A23), estudantes que queiram aprender música ou aprimorar seus conhecimentos (A1, A6, A13, A14, A18, A20, A21, A24 e A25) e adultos (A4 e A12). Em relação à abordagem, o ensino para as crianças é focado na utilização das tecnologias com o intuito de introduzir música, já com os estudantes a abordagem é centrada em despertar o interesse por música ou melhorar o entendimento e nos adultos melhorar o desempenho em uma peça musical principalmente o elemento ritmo.

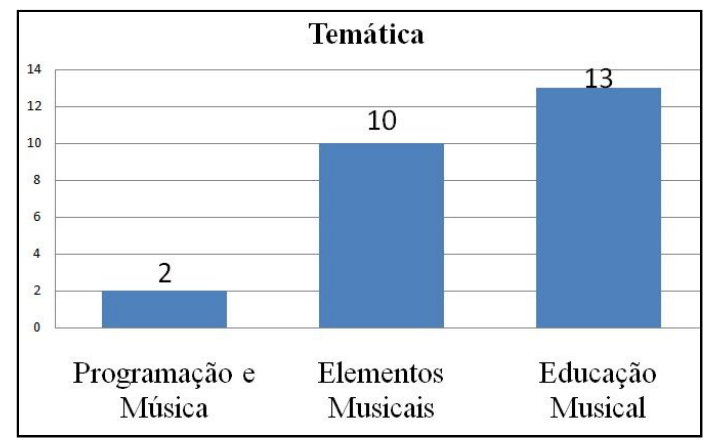

Figura 4. Temáticas encontradas nos artigos 
VIII Congresso Brasileiro de Informática na Educação (CBIE 2019)

Anais do XXX Simpósio Brasileiro de Informática na Educação (SBIE 2019)

\subsubsection{Como os jogos sérios estão sendo utilizados no ensino de Música?}

A partir dos estudos encontrados, é importante destacar os jogos sérios e sua utilização no ensino de Música. O primeiro estudo A1 contém quatro jogos, resultado de uma pesquisa interdisciplinar entre Programação e Música. O primeiro jogo "Drums Listenand Execute Game" foi desenvolvido para melhorar a percepção musical, já o segundo "Keyboard Chord Trainer" tem o objetivo de melhorar a leitura da trilha sonora, o terceiro jogo "Guitar Scale Mastery" tem o objetivo de melhorar as habilidades ao tocar guitarra e o quarto jogo "Sight Reading Game" tem como intuito ajudar pessoas a melhorar suas habilidades no baixo.

O A2 descreve três jogos, o "Harmonic Walk" para ensinar harmonia tonal e harmonização de melodia, "Jazz Improvisation" para ouvir partituras e recomposição musical, "Following the Cuckoo Sound" para ajudar as crianças cegas a andar em linha reta por meio do som. O estudo A3 apresenta o jogo "Game World" que ajuda as crianças a aprender os conhecimentos básicos sobre as teclas do piano e as notas musicais associadas. O A5 descreve o jogo "Flappy Crab" que tem como objetivo trabalhar áreas como a memória auditiva e a discriminação de alturas, abordagem simultânea à simbologia própria da ortografia musical.

Os demais jogos que estão relacionados ao estudo são o jogo "Caklempong" que busca preservar a Música tradicional caklempong na Malásia e ensina a tocar o instrumento canang (A6). O "APP" é um jogo de aprendizagem digital voltado a musicalidade e ritmo (A7). O jogo "Touching Notes" baseia-se em gestos, na qual exige uma movimentação do corpo para tocar as notas musicais (A9). O jogo "Note Code" apresenta um quebra-cabeça de Programação musical projetado como um dispositivo tangível acoplado a uma interface gráfica do usuário GUI (A10). O jogo E-Cecilia contêm exercícios de canto gerados automaticamente, além de um sistema de avaliação do desempenho do usuário (A11). O jogo "Following Puccini" acompanha o desempenho de uma peça musical, este processo é muito importante para os estudantes de Música obterem conhecimentos e habilidades sobre solfejo, harmonia, processos de composição, formas de Música e reconhecimento de timbre (A12). "Tone Deafener" é um jogo baseado em habilidade no qual o jogador controla o jogo ao tocar um instrumento musical acústico, cada nota tocada corretamente é atribuída uma pontuação ao usuário (A13). O "E-Santur" foi desenvolvido para possibilitar o aprendizado de conceitos musicais de Música clássica persa com o instrumento chamado Santur (A14). "Pitch Paradise" é um jogo para auxiliar o ensino de Música às crianças, além de apresentar três mini-jogos sobre ritmo, pitch e melodia (A15). O jogo "Piano Game" utiliza o computador para a educação musical e estimula as atividades de audição, execução e melhora o reconhecimento das notas musicais (A17). O "Cha-Luva Swing Festival" é um jogo com o objetivo pedagógico geral de estimular a curiosidade e a criatividade dos jogadores, permitindo que eles facilmente reproduzam Música usando gamepads como instrumentos musicais (A18).

O jogo "TuneTrain" foi desenvolvido para a capacitar crianças a compor Música sem a necessidade de ensinar conceitos musicais explicitamente (A19). "Chorlody" é um jogo de ação rítmica que ajuda os alunos a desenvolverem habilidades para reconhecer progressão de acordes, este jogo mostra os acordes para ajudar na cognição auditiva dos jogadores e apresenta diferentes combinações de notas (A20). "Guitar 
man" é um jogo com o objetivo de alcançar a cooperação musical e trabalhar performances com alguns instrumentos musicais por meio de computação gráfica (A21). O "GenVirtual" é um jogo musical que possibilita seguir uma sequência de cores e sons emitidos a partir dos objetos virtuais projetados no mundo real (A22).

Outra abordagem encontrada foi à utilização do jogo "Zorelha" no qual os sons dos instrumentos são aleatoriamente executados e a criança deve indicar o instrumento em execução clicando no personagem (A23). O jogo "Musikinésia" auxilia alunos dos cursos de Música na identificação das teclas de um teclado musical, além de ensinar outros conceitos musicais básicos, como andamentos, acidentes e leitura musical (A24). O "Descobrindo Sons" é um jogo de escuta, em que o jogador/aluno deve prestar atenção ao som tocado e tentar adivinhar, pelo timbre, qual instrumento está emitindo o som (A25). Os artigos A4, A8 e A16 não relataram nenhum jogo sério por se tratarem de estudos voltados para métodos e design de jogos digitais para o ensino de Música.

Desses jogos, observa-se que 24 dos 25 estudos foram direcionados para uma experiência primária com Música, apenas o artigo A12 requer que o usuário tenha conhecimentos sobre Música. Além disso, 20 artigos utilizaram os jogos para melhorar a percepção musical com estímulo à audição, promovendo ensinamentos a respeito de elementos musicais (ritmo, timbre, melodia, harmonia, altura e solfejo) e 5 artigos usam os jogos para ensinar conhecimentos básicos com uma abordagem a instrumentos de culturas locais e leitura de partituras (Figura 5).

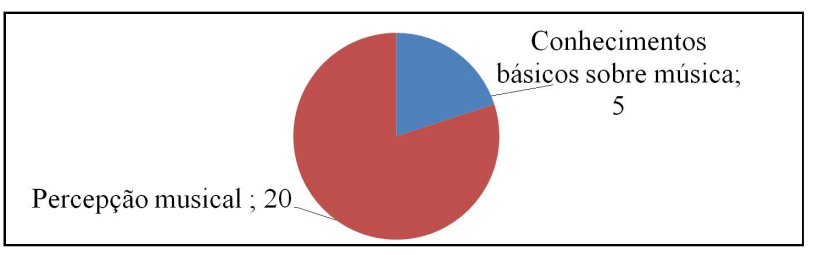

Figura 5. Utilização dos jogos sérios no ensino de Música

\section{Considerações Finais}

O uso de jogos sérios para o ensino de Música é considerado uma área relevante de pesquisa na Informática na Educação, seja para o público infantil ou adulto. Os resultados mostraram que há um intuito comum entre tais aplicações que é o de ensinar diversos assuntos de Música. Diversas pesquisas se propuseram a ensinar, mas para o ensino-aprendizagem de Programação e Música o número de artigos obtidos nos resultados da RS foram apenas dois. Com isso, é possível observar que a maioria buscou ensinar em um contexto voltado a educação musical abordando elementos como ritmo, timbre, melodia, harmonia, altura, solfejo e o principal público-alvo foram crianças. No entanto, os adultos foram pouco explorados no ensino-aprendizagem por meio de jogos sérios.

Com a análise dos dados extraídos, foi possível observar duas iniciativas sobre jogos com o ensino interdisciplinar de Música e Programação, divulgadas nas bases. Portanto, os resultados obtidos na RS serviram de análise para afirmar que há uma carência de jogos desenvolvidos para ensino de Música, no sentido de apoio ao ensino de disciplinas. Sendo assim, propõe-se como trabalho futuro o desenvolvimento de um jogo para suprir a necessidade de apoio ao ensino de Música e Programação, levando 
VIII Congresso Brasileiro de Informática na Educação (CBIE 2019)

Anais do XXX Simpósio Brasileiro de Informática na Educação (SBIE 2019)

em conta o grande desafio de ingressar um músico na área da Computação, ter um diferencial no mercado de trabalho e conseguir desenvolver novas tecnologias para a área de Música.

\section{Referências Bibliográficas}

Araújo, Doraci Modesto de Pinho.; Moreno, J. C. O ensino da música na escola : desafios para o professor. Paraná, 2009.

Battistela, P.E..;Wangenheim, C.G. von; Fernandes, J.M. Como jogos educacionais são desenvolvidos? Uma revisão sistemática da literatura. In: XXII Workshop sobre Educação em Computação (WEI 2014). Sociedade Brasileira de Computação, 2014.

Biolchini, J.; Mian, P. G.; Natali, A. C. C.; Travassos, G. H. Systematic review in software engineering. Technical report, RT-ES 679/05 System Engineering and Computer Science Dept., COOPE/UFRJ. 2005.

Cox, K. K.; Bittencourt, R.A.;Estudo Bibliográfico sobre o Processo de Construção de Jogos Digitais: A Necessidade de Sinergia entre o Educar e o Divertir. Revista Brasileira de Informática na Educação, v. 25, n. 1, 2017.

Crocco, Laura; Madill, Catherine J.; Mccabe, Patricia. Evidence-based frameworks for teaching and learning in classical singing training: A systematic review. JournalofVoice, v. 31, n. 1, p. 130. e7-130. e17, 2017.

Fernandes, Sandra Gomes; Coutinho, Clara Pereira. Tecnologias no Ensino da Música: revisão integrativa de investigações realizadas no Brasil e em Portugal. Educação, Formação \& Tecnologias-ISSN 1646-933X, v. 7, n. 2, p. 94-109, 2014.

Freitas, V. C. B.; Adamatti, D. Educa Direito: um jogo sério para o ensino de direito do trabalho. Revista Brasileira de Informática na Educação, v. 24, n. 3, 2016.

Kitchenham, Barbara. Procedures for performing systematic reviews. Keele, UK, Keele University, v. 33, n. 2004, p. 1-26, 2004.

Moreira, E. E.; Castro, A. B. B. Jogos Digitais Como Objeto de Aprendizagem. $5^{\text {a }}$ Jornada Científica e Tecnológica da FATEC de Botucatu. Botucatu-São Paulo, 2016.

Pocinho, M. Lições de revisão sistemática e metanálise, 2008. Disponível em: $<\mathrm{http}$ ://docentes.ismt.pt/ m_pocinho/Licoes_de_revisao_sistematica_e_metanalise.p df>. Acesso em: 12 Mar. 2018.

Raziūnaite, P. et al. Designing an educational music game for digital game based learning: A Lithuanian case study. In: 2018 41st International Convention on Information and Communication Technology, Electronics and Microelectronics (MIPRO). IEEE, 2018. p. 0800-0805.

Salen, K.; Tekinbaş, K. S.; Zimmerman, E. Rules of Play: Game Design Fundamentals. MIT press, 2004.

Sarinho, V. T. LibrasZap - Um Jogo Baseado em Mensagens Instantâneas para Avaliação de Conhecimentos na Língua Brasileira de Sinais. Revista Brasileira de Informática na Educação, v. 25, n. 1, 2017. 